\title{
Conservative rehabilitation for a patient presenting with severe orthostatic hypotension after surgical management of brainstem tumor: illustrative case
}

\author{
Misato Nagumo, MD, ${ }^{1}$ Syoichi Tashiro, MD, PhD, ${ }^{1,2}$ Nanako Hijikata, MD, ${ }^{1}$ Aiko Ishikawa, MD, PhD, ${ }^{1}$ Takenori Akiyama, MD, PhD, ${ }^{3}$ \\ and Tetsuya Tsuji, MD, $\mathrm{PhD}^{1}$
}

Departments of ${ }^{1}$ Rehabilitation Medicine and ${ }^{3}$ Neurosurgery, Keio University School of Medicine, Tokyo, Japan; and ${ }^{2}$ Department of Rehabilitation Medicine, Kyorin University School of Medicine, Tokyo, Japan

\begin{abstract}
BACKGROUND Blood pressure (BP) dysregulation is frequently observed in patients after surgical management of brainstem lesions; however, there has been no standard rehabilitation. Considering the conflicting risks for hypoperfusion and disuse syndrome in these patients, a safe and effective rehabilitative strategy is warranted.

OBSERVATIONS A 50-year-old man who had undergone craniotomy for resection of a recurrent dorsal medullary epidermoid cyst developed persistent orthostatic hypotension. It was resistant to physical exercise, pharmacological therapy, abdominal binders, and compression stockings; therefore, it inhibited postoperative rehabilitation. Although the responsible lesion was not clearly visible on the postoperative image, accompanying symptoms, including segmental sensory impairment, implied an improvement in BP control. Although there was a trade-off between the risk of developing disuse syndrome and a delay in functional recovery, the authors decided to continue a conservative rehabilitation strategy rather than increasing the workload. The patient's BP control was gradually restored by the seventh postoperative week, and the authors proceeded with basic activity training.

LESSONS A conservative prognostic prediction-based rehabilitation strategy was applied in this case. The precise evaluation of the accompanying neurological symptoms was helpful in deciding the treatment regimen. The conflicting risks for hypoperfusion and disuse syndrome in such cases must be considered.
\end{abstract}

https://thejns.org/doi/abs/10.3171/CASE2136

KEYWORDS postoperative rehabilitation; surgery; tumors; cerebellar ataxia; autonomic; segmental sensory impairment

Neurogenic orthostatic hypotension $(\mathrm{OH})$ frequently occurs after surgical removal of dorsal medullary tumors. In patients with brainstem lesions, $\mathrm{OH}$ may develop because of the interruption of descending sympathoexcitatory pathways from the neurons of the ventrolateral medulla (VLM). ${ }^{1}$ Despite successful surgery for dorsal medullary tumor and medical treatment, $\mathrm{OH}$ often lasts approximately 3 to 6 months $^{2-6}$ and can inhibit orthostatic activities and rehabilitation. In addition, according to a literature review, 6 of 15 such patients die from complications related to $\mathrm{OH}$ and brainstem compression. ${ }^{7}$ However, to the best of our knowledge, there has been no previously established rehabilitation strategy for attenuating the symptoms of $\mathrm{OH}$ to prevent postoperative hypoperfusion in cases with brainstem lesions. ${ }^{8,9}$ Here, we describe the postoperative conservative rehabilitation of a patient who was unable to stand up for $>7$ weeks because of $\mathrm{OH}$ after craniotomy for resection of a dorsal medullary epidermoid cyst. It was helpful for predicting the prognosis of $\mathrm{OH}$ and planning a rehabilitative strategy to evaluate the recovery process of postoperative symptoms, such as segmental sensory impairment and ataxia.

\section{Illustrative Case}

A 50-year-old man who had undergone craniotomy in 1998, 2009, 2012, and 2014 presented with progressively exacerbated dysphagia, disorders of gait, and coordinated movement of the upper extremities, indicating recurrence of a dorsal medullary epidermoid cyst. He was

ABBREVIATIONS BP = blood pressure; MEP = motor evoked potential; MRI = magnetic resonance imaging; OH = orthostatic hypotension; RVLM = rostral VLM; $\mathrm{VLM}=$ ventrolateral medulla.

INCLUDE WHEN CITING Published June 21, 2021; DOI: 10.3171/CASE2136.

SUBMITTED January 17, 2021. ACCEPTED April 7, 2021.

(C) 2021 The authors, CC BY-NC-ND 4.0 (http://creativecommons.org/licenses/by-nc-nd/4.0/). 
admitted to the hospital for craniotomy in 2019. Before admission, he had been walking with the aid of a right Lofstrand crutch and had been able to commute for 30 minutes by train. He was able to perform his daily activities. He ate ordinary meals, although he had slight difficulty in swallowing. Magnetic resonance imaging (MRI) showed an epidermoid cyst on the inferior surface of the left cerebellar hemisphere extending into the lower medulla oblongata (Fig. $1 A$ and $B$ ). The tumor was almost totally resected during surgery; it was removed from the surface of the brainstem as much as possible, leaving only the epithelium (Fig. 1C and D). The patient developed temporary bradycardia and decreased motor evoked potentials (MEPs) of the right upper extremity during surgical manipulation of the left dorsal side wall of the tumor capsule. After surgery, he was admitted to the intensive care unit. He could breathe spontaneously after the surgery; however, ventilator management and administration of noradrenaline were needed because of sleep apnea, loss of swallowing reflex, and low blood pressure (BP). He was referred to the rehabilitation department on postoperative day 2 for treatment. The ventilator was no longer needed on postoperative day 7 because of the disappearance of sleep apnea. However, a tracheostomy was performed to manage the swallowing dysfunction.

After the noradrenaline level was tapered off on postoperative day 2, he developed $\mathrm{OH}$. His BP dropped from 136/61 $\mathrm{mm} \mathrm{Hg}$ in the supine position to $73 / 45 \mathrm{~mm} \mathrm{Hg}$ in the sitting position; his heart rate remained unchanged (75 beats per minute). Performing lower extremity exercise while sitting increased BP to $84 / 52 \mathrm{~mm} \mathrm{Hg}$; however, his systolic BP dropped to $50 \mathrm{~mm} \mathrm{Hg}$ after standing, and he felt dizzy and nauseous. On postoperative day 13, an oral dose of $10 \mathrm{mg}$ of amezinium metilsulfate was administered three times a day as first-line treatment, but it did not induce any remarkable result. On postoperative day $23,100 \mathrm{mg}$ of droxidopa was administered three times a day, and the number of doses was increased to four times a day (total $400 \mathrm{mg}$ ) on postoperative day 29. Simultaneously, the use
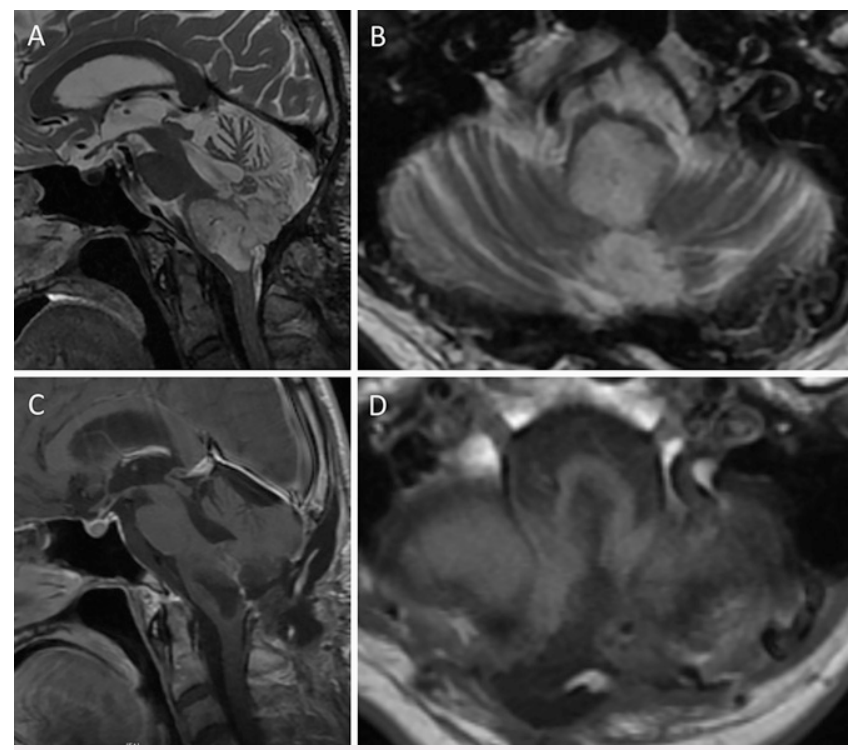

FIG. 1. A: Preoperative sagittal T2-weighted MRI with gadoliniumbased contrast agent showing the location of the lesion in the lower medulla oblongata. B: Preoperative axial T2-weighted MRI showing the tumor compressing normal tissue. C: Postoperative sagittal T1-weighted MRI using gadolinium-based contrast agent. D: Postoperative axial T1-weighted MRI showing the tumor after near-total resection. of elastic bandages for the legs and abdominal bandages also did not yield positive results. We continued with daily nonintensive training composed of a wheelchair transfer motion and sitting exercises with and without back support until systolic BP dropped to $80 \mathrm{~mm} \mathrm{Hg}$ or symptoms appeared. Additionally, we continued with extremitystrengthening exercises in the supine position to prevent deconditioning at every rehabilitation session.

On postoperative day 13 , cranial nerve examination revealed horizontal directional nystagmus and decreased tongue movement speed. Other neurological symptoms included mild to moderate bilateral ataxia, which was most prominent in the left upper extremity, and segmental sensory impairment. Tactile, pain, and temperature sensations were moderately to severely impaired below the $\mathrm{T7}$ level on the left side. Position sense was impaired severely on the right side of the extremities and moderately on the left side (Table 1).

On postoperative day 28 , ataxia of the upper extremity as confirmed using the finger-to-nose test was improved to a mild level. Tactile, pain, and temperature sensations improved to normal levels below the $\mathrm{T} 7$ level on the left side of the trunk and to moderate levels in the left lower extremity. Position sense improved to a mild to normal level in the left extremities, although the severity of impairment did not change significantly on the right side (Table 1). With these improvements, the patient's systolic BP became higher than $90 \mathrm{~mm} \mathrm{Hg}$ while performing lower extremity exercise in the sitting position around postoperative day 30 . The gradual improvement of these neurological symptoms along with better BP control implied an improvement in $\mathrm{OH}$. We decided not to start tilt-up table training and in the meanwhile extended the duration of sitting on the side of the bed or in a reclining wheelchair. At that point, we judged that the tilt training might trigger hypoperfusion because the tolerable time duration for orthostatic posture was still short, the body fixation apparatus of the tilt-up table restricted lower extremity exercise, and a quick BP decline needing an immediate postural change was sometimes observed.

TABLE 1. SARA and segmental sensory impairment on postoperative days 13 and 28

\begin{tabular}{cccccc}
\hline & \multicolumn{2}{c}{ Day 13 } & & \multicolumn{2}{c}{ Day 28 } \\
\cline { 2 - 3 } \cline { 5 - 6 } & $\mathrm{Rt}$ & $\mathrm{Lt}$ & & $\mathrm{Rt}$ & $\mathrm{Lt}$ \\
\hline SARA & & & & \\
\hline Finger-to-nose test & 2 & 3 & & 2 \\
\hline Heel-to-shin slide & 1 & 2 & & 1 & 2 \\
\hline $\begin{array}{c}\text { Segmental sensory } \\
\text { impairment }\end{array}$ & & & & \\
\hline $\begin{array}{c}\text { Tactile, pain, \& } \\
\text { temperature sense }\end{array}$ & & & & & \\
\hline Upper extremity & Normal & Normal & Normal & Normal \\
\hline Body trunk & Normal & Moderate & Normal & Normal \\
\hline Lower extremity & Normal & Severe & Normal & Moderate \\
\hline Position sense & & & & \\
\hline Upper extremity & Severe & Moderate & Severe & Normal \\
\hline Lower extremity & Severe & Moderate & Severe & Mild \\
\hline
\end{tabular}

SARA = scale for the assessment and rating of ataxia, Mod $=$ moderate, temp $=$ temperature. 
Although $\mathrm{OH}$ persisted, dysphagia improved gradually over time. Swallowing training was initiated on postoperative day 10 after tracheostomy. The patient was given soft meals on postoperative day 30 after the videofluorographic swallowing study revealed no severe deficit. The tracheal cannula was removed on postoperative day 35. As for bladder function, the symptoms of an overactive bladder were observed after the urinary catheter was removed on postoperative day 27 ; the symptoms gradually improved with mirabegron, which is a $\beta_{3}$-adrenoceptor agonist.

As we expected, $\mathrm{OH}$ improved 4 months postoperatively, and the drug doses were reduced; the patient received $15 \mathrm{mg}$ of amezinium metilsulfate once daily and $100 \mathrm{mg}$ of droxidopa thrice daily. The severity of ataxia and tactile, pain, and temperature sensations did not change significantly. The position sense in the right extremities improved to a mild to moderate level. The patient walked without syncopal episodes and maintained a BP of 100 to $120 / 70 \mathrm{~mm}$ $\mathrm{Hg}$ while performing daily life activities.

\section{Discussion}

\section{Observations}

We are the first to report the rehabilitation trajectory for a patient presenting with severe $\mathrm{OH}$ after surgical management of a brainstem tumor as well as the goal of rehabilitation along the recovery process of brainstem function. We believe that this study will help to predict the rehabilitation trajectory in similar cases after neurosurgery. To the best of our knowledge, there have been no reported cases of segmental sensory impairment after brainstem surgery, although there are a few reports on stroke. ${ }^{10}$

In the present case, a close investigation of neurological symptoms indicated that the lesion was most likely to be at the lower level of the medulla oblongata. Before surgery, the patient had presented with only the exacerbation of ataxia and dysphagia with the growth of the tumor; he had no syncopal episode. The patient developed ataxia most likely because of compression of the left side of the cerebellum, inferior cerebellar peduncle, spinocerebellar tract, cuneate nucleus, and gracile nucleus by the tumor; the pathogenesis of ataxia was de- rived from cerebellar and sensory origin. Dysphagia was presumed to occur because of the compression of the solitary nucleus, ambiguous nucleus, and swallowing-related neurons in the bulbar reticular formation by the tumor. After surgery, in addition to further exacerbation of the existing symptoms, the patient presented with $\mathrm{OH}$, apnea, and segmental sensory impairment, which were probably caused by transient edema or ischemia around the area compressed by the tumor.

The sensory fibers mediating tactile, pain, and temperature sensations from the lower extremity ascend the lateral part of the lateral spinothalamic tract and pass through the medulla oblongata. ${ }^{11}$ Therefore, the postoperative lesion was expected to extend from the lateral part of the right lateral spinothalamic tract to both the cuneate nucleus and gracile nucleus. In addition to this area, the lesion probably extended to the left side, as expected from the decrease in MEPs during surgery (Fig. 2).

Although apnea and swallowing function gradually improved, $\mathrm{OH}$ persisted and inhibited rehabilitation. The rostral VLM (RVLM) is an important sympathetic nervous system in the regulation of BP that provides atonic retention to peripheral vessels. ${ }^{12}$ It is known that the solitary nucleus inhibits the RVLM via the caudal VLM; therefore, the dysfunctional solitary nucleus facilitates RVLM activation, causing central hypertension. In our patient, who did not present with supine hypertension, $\mathrm{OH}$ was possibly caused by disturbance of not only the solitary nucleus but also the descending sympathoexcitatory pathways from the RVLM. Because the lesion probably included both sides of the solitary nucleus and descending sympathoexcitatory pathways, $\mathrm{OH}$ persisted and was resistant to treatment.

$\mathrm{OH}$ substantially restricts patients in proceeding with rehabilitation as well as functional independence. Hypoperfusion in the central nervous system may result in weakness, fatigue, palpitation, dizziness, nausea, musculoskeletal pain, vision disorder, cognitive impairment, syncope, ischemic disease, and fall. ${ }^{13-16} \mathrm{~A}$ systematic review summarizes various methods for the management of $\mathrm{OH}$, such as exercise, functional electrical stimulation, compression, physical counter-maneuvers, compression, sleeping with the head raised, and dietary measures (water or food intake). ${ }^{8,9}$ However, there has

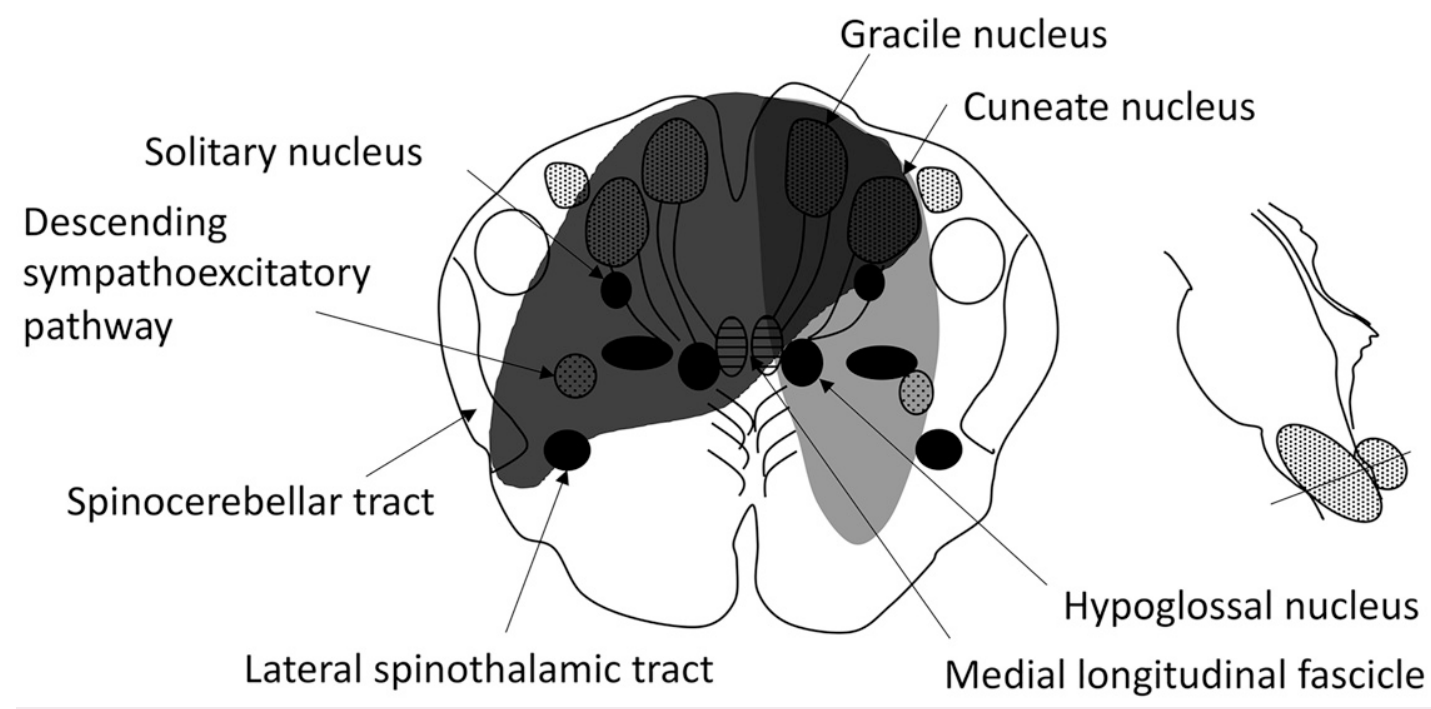

FIG. 2. An illustration of the postoperative lesion presumed at the lower level of the medulla oblongata. 
been no consensus on a rehabilitation strategy for $\mathrm{OH}$ after neurosurgery. This case suggests that a conservative strategy instead of proceeding with tilt-up table habituation is applicable depending on the situation, such as the postoperative period. We expected an improvement in the function of the medulla oblongata, especially BP regulation, because the improvement of the segmental sensory disorder and ataxia indicated a reduction in lesion size. Besides, there were potential risks of hypoperfusion for the tilt-up table training because of the short tolerable time duration for orthostatic posture, the restriction of lower extremity exercise on the tilt-up table, and the existence of a sudden BP decline. Overall, we did not proceed with excessive training when pharmacological and nonpharmacological treatments were ineffective. Although the strategy worked in this case, it may not be equally applicable to all cases with $\mathrm{OH}^{9}$ There are reports of a successfully applied unique tilt-table device with the function of passive stepping or functional electrical stimulation ${ }^{17,18}$ that might have served in the present case. Above all, it is imperative not to cause deconditioning because of selecting a conservative strategy.

\section{Lessons}

$\mathrm{OH}$ may substantially interfere with postoperative rehabilitation, causing secondary damage due to cerebral hypoperfusion or musculoskeletal injury due to falls. The periodic neurological examination of related symptoms may be useful to predict functional prognosis, even if the lesion causing $\mathrm{OH}$ is unclear on brain images. A conservative prognostic prediction-based rehabilitation strategy that considers the conflicting risks of developing hypoperfusion from moving and disuse syndrome from immobility could be applicable to cases similar to the present one. Given that appropriate rehabilitation methods for patients with impaired brainstem function are limited, an accumulation of cases is required.

\section{Acknowledgments}

This work was supported by a general research fund (M.N., S.T.) from the Department of Rehabilitation Medicine, Kyorin University School of Medicine.

We thank Drs. Asaka Minami and Kyohei Juumi from the Department of Rehabilitation Medicine, Keio University School of Medicine, for their sincere assistance and advice in the treatment of the present case.

\section{References}

1. Arango V, Ruggiero DA, Callaway JL, et al. Catecholaminergic neurons in the ventrolateral medulla and nucleus of the solitary tract in the human. J Comp Neurol. 1988;273(2):224-240.

2. Hocker S, Hoover JM, Puffer RC, Meyer FB. Orthostatic hypotension following resection of a dorsal medullary hemangioblastoma. Neurocrit Care. 2012;16(2):306-310.

3. Idiaquez J, Araya P, Benarroch E. Orthostatic hypotension associated with dorsal medullary cavernous angioma. Acta Neurol Scand. 2009;119(1):45-48.

4. Jabary NS, Sarabia R, Sanchez T, Gordillo R. Midodrine treatment in the management of severe orthostatic hypotension after hemangioblastoma surgery. Acta Neurochir (Wien). 2007;149(3):303-306.

5. Tadros R, Tonkin A. Bilateral bulbar palsy and postural hypotension following surgery for fourth ventricle subependymoma. J Clin Neurosci. 2009;16(12):1646-1648.
6. Yamashita A, Hirayama M, Koike Y, et al. Orthostatic hypotension caused by a localised dorsal medullary tumour. J Neurol Neurosurg Psychiatry. 1996;61(1):118-119.

7. Nangunoori R, Koduri S, Singh A, Aziz K. Refractory orthostatic hypotension caused by a recurrent hemangioblastoma: case report and review of the literature. Cureus. 2016;8(3):e540.

8. Eschlböck S, Wenning G, Fanciulli A. Evidence-based treatment of neurogenic orthostatic hypotension and related symptoms. J Neural Transm (Vienna). 2017;124(12):1567-1605.

9. Mills PB, Fung CK, Travlos A, Krassioukov A. Nonpharmacologic management of orthostatic hypotension: a systematic review. Arch Phys Med Rehabil. 2015;96(2):366-375.e6.

10. Yamamoto R, Nishiyama T, Kurokawa $T$, et al. A case of cavernous angioma in the lower pons, showing subacute onset of unilateral cranial nerve palsy and segmental sensory disturbance. Article in Japanese. Rinsho Shinkeigaku. 2009;49(1):32-36.

11. Matsumoto S, Okuda B, Yamasaki M, et al. Segmental sensory disturbance in brain stem infarctions of the lateral lower pons and lateral medulla-clinico-anatomical evaluation by MRI. Article in Japanese. Rinsho Shinkeigaku. 1987;27(3):350-355.

12. Martín-Gallego A, Andrade-Andrade I, Dawid-Milner MS, et al. Autonomic dysfunction elicited by a medulla oblongata injury after fourth ventricle tumor surgery in a pediatric patient. Auton Neurosci. 2016;194:52-57.

13. Bradley JG, Davis KA. Orthostatic hypotension. Am Fam Physician. 2003;68(12):2393-2398.

14. Heims HC, Critchley HD, Martin NH, et al. Cognitive functioning in orthostatic hypotension due to pure autonomic failure. Clin Auton Res. 2006;16(2):113-120.

15. Mol A, Bui Hoang PTS, Sharmin S, et al. Orthostatic hypotension and falls in older adults: a systematic review and meta-analysis. J Am Med Dir Assoc. 2019;20(5):589-597.e5.

16. Noda K, Nakajima S, Sasaki F, et al. Middle cerebral artery occlusion presenting as upper limb monochorea. J Stroke Cerebrovasc Dis. 2015;24(10):e291-e293.

17. Jacobs PL, Johnson B, Mahoney ET. Physiologic responses to electrically assisted and frame-supported standing in persons with paraplegia. J Spinal Cord Med. 2003;26(4):384-389.

18. Luther MS, Krewer C, Müller F, Koenig E. Comparison of orthostatic reactions of patients still unconscious within the first three months of brain injury on a tilt table with and without integrated stepping. A prospective, randomized crossover pilot trial. Clin Rehabil. 2008;22(12):1034-1041.

\section{Disclosures}

The authors report no conflict of interest concerning the materials or methods used in this study or the findings specified in this paper.

\section{Author Contributions}

Conception and design: Tashiro, Nagumo. Acquisition of data: Nagumo, Ishikawa. Analysis and interpretation of data: Nagumo, Ishikawa, Akiyama. Drafting the article: Tashiro, Nagumo, Hijikata, Akiyama. Critically revising the article: Nagumo, Ishikawa, Akiyama, Tsuji. Reviewed submitted version of manuscript: Tashiro, Tsuji. Approved the final version of the manuscript on behalf of all authors: Tashiro. Study supervision: Tsuji. Secured funding: Tashiro.

\section{Correspondence}

Syoichi Tashiro: Keio University School of Medicine, Tokyo, Japan. s-tashiro@z2.keio.jp. 